\title{
Prophylactic effect of rectal indomethacin plus nitroglycerin administration for preventing pancreatitis after endoscopic retrograde cholangiopancreatography in female patients
}

\author{
Yunfeng Wang ${ }^{1 \#}$, Bin Xu ${ }^{1 \#}$, Wenzhong Zhang ${ }^{1}$, Jie Lin ${ }^{1}$, Gang Li ${ }^{1}$, Wei Qiu ${ }^{1}$, Yueming Wang ${ }^{1}$, Duo Sun ${ }^{2}$, \\ Yongbing Wang ${ }^{1}$ \\ ${ }^{1}$ Department of General Surgery, Pudong New Area People's Hospital, Shanghai University of Medicine \& Health Sciences, Shanghai, China; \\ ${ }^{2}$ Department of General Surgery, Shenyang Fourth People's Hospital, Shenyang, China \\ Contributions: (I) Conception and design: Y Wang, B Xu; (II) Administrative support: D Sun, Y Wang; (III) Provision of study materials or patients: \\ W Zhang, J Lin, W Qiu; (IV) Collection and assembly of data: Y Wang, B Xu, Y Wang; (V) Data analysis and interpretation: Y Wang, Y Wang; (VI) \\ Manuscript writing: All authors; (VII) Final approval of manuscript: All authors. \\ \#These authors contributed equally to this work. \\ Correspondence to: Yongbing Wang, MD. Department of General Surgery, the Affiliated Pudong New Area People's Hospital of Shanghai Health \\ Medical College, No. 490, Chuanhuan South Road, Pudong New Area, Shanghai, China. Email: 2489414597@qq.com.
}

Background: Prophylactic pancreatic stent placement (PSP) and rectal indomethacin suppository are recommended to prevent post-endoscopic retrograde cholangiopancreatography (ERCP) pancreatitis (PEP) in high-risk cases. Clinical trials on the use of nitroglycerin to reduce PEP have reached no definitive conclusion. Our study aimed to determine whether treatment with rectal indomethacin plus nitroglycerin could eliminate the need for PSP in patients.

Methods: In this randomized clinical trial, patients were allocated into groups using a random number table, with each patient receiving a pre-made envelope containing their intervention prior to ERCP. The three treatment groups were: the placebo group, the indomethacin + nitroglycerin group, and the PSP group. The subjects were assessed for PEP and its severity by a panel of independent and blinded adjudicators.

Results: A total of 526 patients were eligible for inclusion. The placebo group included 176 patients, the indomethacin + nitroglycerin group included 176 patients and the PSP group included 174.A diagnosis of PEP was made in $64(12.2 \%)$ cases. The rate of PEP in the three study groups placebo group, indomethacin + nitroglycerin group and the PSP group was $19.3 \%, 5.1 \%$, and $12.1 \%$, respectively.

Conclusions: The risk of post-ERCP pancreatitis in the indomethacin + nitroglycerin group was $7 \%$ lower than that in the PSP. Indomethacin + nitroglycerin is superior to PSP in preventing and relieving the severity of post-ERCP pancreatitis in patients with difficult intubation. Indomethacin plus nitroglycerin can avoid the need for PSP in the prevention of post-ERCP pancreatitis.

Trial registration: Current Controlled Trials ChiCTR2000033944.

Keywords: Hyperamylasemia; indomethacin; nitroglycerin; resistin; pancreatic stent; endoscopic retrograde cholangiopancreatography (ERCP)

Submitted Sep 07, 2020. Accepted for publication Nov 12, 2020.

doi: $10.21037 /$ apm-20-1963

View this article at: http://dx.doi.org/10.21037/apm-20-1963 


\section{Introduction}

Post-endoscopic retrograde cholangiopancreatography (ERCP) pancreatitis (PEP) is the most common postoperative adverse event in patients who undergo ERCP. The incidence of PEP ranges between 2-4\%, although in some high-risk patients it is as high as $8-20 \%$ (1). PEP is associated with increased morbidity and mortality as well as higher health care costs (2). The risk factors for PEP need to be recognized in order to develop and improve prevention strategies, which include careful selection of ERCP patients and appropriate mechanical and pharmacological preventive measures.

Currently, the main clinical approaches to preventing acute PEP are drugs and pancreatic stent placement (PSP) (3-7). However, studies have reported that, while PSP can reduce the incidence of PEP a certain extent, the universal applicability of this treatment is limited by the substantial economic burden it places on patients. Consequently, a number of studies have evaluated the efficacy of pharmacologic prophylaxis in the prevention of PEP (8-13). In high-risk patients, several measures can be undertaken to limit the risk of PEP, including administration of rectal nonsteroidal anti-inflammatory drugs, prophylactic placement of pancreatic duct stents, and liberal administration of lactated Ringer solution. Metaanalysis of all pharmacologic prophylaxis, randomized, controlled trials noted reduced PEP rates in both averageand high-risk patients. However, these studies are not entirely conclusive given the inherent limitations related to the select study methodology.

Rectal nonsteroidal anti-inflammatory drugs (NSAIDs) have been investigated in multiple randomized controlled trials for reducing the incidence of PEP (14-17). NSAIDs are believed to limit the risk of developing acute pancreatitis via the inhibition of inflammatory mediators that trigger a complex cascade of inflammatory events; this is a critical process in the pathogenesis of pancreatitis. Moreover, nitroglycerin, nifedipine, and other drugs can reduce pressure on the sphincter of Oddi (18-20). Previously, indomethacin and diclofenac were used in combination to prevent postoperative pancreatitis. This article is the first to use indomethacin and nitroglycerin to prevent postoperative pancreatitis.

Given the high financial costs and potential disadvantages associated with PSP, minimizing its use in patients who undergo ERCP could result in major clinical and economic benefits. Therefore, this study aimed to determine whether rectal indomethacin plus nitroglycerin could reduce the need for PSP in patients with who underwent ERCP.

We present the following article in accordance with the CONSORT reporting checklist (available at http://dx.doi. org/10.21037/apm-20-1963).

\section{Methods}

\section{Patients}

Between January 2017 and December 2019, 600 patients who underwent ERCP in Shanghai Pudong New Area People's Hospital were enrolled in this randomized clinical trial. The minimum sample size was calculated using the formula $\mathrm{n}=\Psi 2\left(\sum \operatorname{si} 2 / \mathrm{k}\right) /\left[\sum(\bar{X} \mathrm{i}-\bar{X}) 2 /(\mathrm{k}-1)\right]$, where $\mathrm{k}$ is the number of groups in the study, $\bar{X} \mathrm{i}$, si is the valuation of the mean and standard deviation of each group, an $\bar{X}=\sum \bar{X} \mathrm{i} / \mathrm{k}, \Psi$ is the bound value, which can be obtained from the $\Psi$ value table. The results showed that more than 150 cases had statistical differences. The inclusion criteria were: female patients aged 20-80 years. Patients were clearly diagnosed with choledocholithiasis by abdominal computed tomography (CT) or magnetic resonance cholangiopancreatography (MRCP) examination before the procedure; preoperative serum amylase level was in the normal range (hospital laboratory reference value, 30-110 U/L); no abdominal pain, fever or other clinical symptoms, or acute inflammation has been conservatively treated and controlled. All procedures performed in this study involving human participants were in accordance with the Declaration of Helsinki (as revised in 2013). The study was approved by Ethics Committee of the Pudong New Area People's Hospital, Shanghai University of Medicine \& Health Sciences (Shanghai, China) (No. 2017-26). Written informed consent was obtained from all patients. Patients with at least one of the following factors for difficult intubation were eligible for inclusion in the study: (I) intraoperative intubation time $>10 \mathrm{~min}$; (II) more than five attempts at cannulation; (III) the papillary sphincter was pre-cut sphincterotomy (PST); (IV) Endoscopic sphincterotomy (EST)/balloon dilatation (EPBD); (V) the guide wire was placed into the pancreatic duct more than once; (VI) contrast agent was inserted into the pancreatic duct.

The exclusion criteria were: (I) pregnant and lactating women; (II) patients with severe cardio-cerebrovascular disease; anemia; kidney disease, or a history of glaucoma and increased intracranial pressure; (III) patients with a 


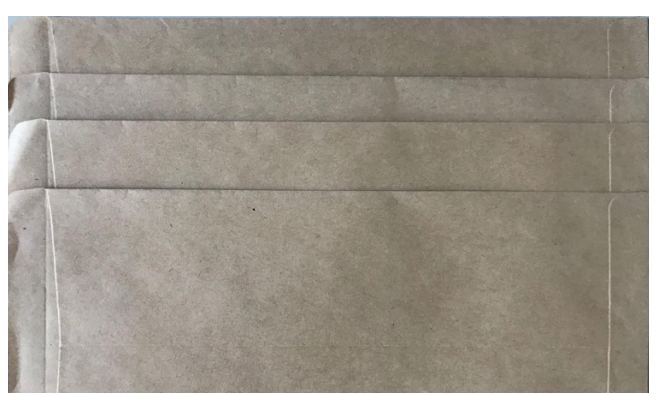

Figure 1 Pre-made envelopes containing the allocation intervention.

history of liver cirrhosis, upper gastrointestinal ulcer, or upper gastrointestinal bleeding; (IV) patients with allergies to contrast agents or unable to tolerate somatostatin or nitroglycerin; (V) patients with mental disorders or illnesses; (VI) patients taking pancreatic toxic drugs (e.g., estrogen, azathioprine, mesalazine, morphine derivatives, or prednisone) or angiotensin receptor blockers; (VII) smokers.

\section{Research methods}

Pre-made envelopes containing the allocation intervention and study number were assigned to the patients $30 \mathrm{~min}$ before the operation to ensure randomization and allocation concealment until administration of the interventions (indomethacin + nitroglycerin, placebo suppository + tablet, and PSP with placebo suppository + tablet). Indomethacin suppositories and placebo suppositories, and nitroglycerin and placebo tablets, which were identical in appearance, were placed in an envelope and sorted sequentially according to the randomization schedule (Figure 1). The patients, residents, and researchers were blinded to the treatment assigned to each participant; only the endoscopist(s), endoscopy nurse, and technician/assistant were aware of the allotted treatments.

All patients received intravenous piperidine $50 \mathrm{mg}$, diazepam $5 \mathrm{mg}$, and anisodamine $10 \mathrm{mg} 10-15 \mathrm{~min}$ before ERCP was performed. The procedure was carried out using an Olympus JF-260V electron duodenoscope. Randomization was carried out on a 1:1 basis using a webbased central randomization system to ensure treatment balance. Indomethacin suppositories were administered by an endoscopy nurse, technician, or the endoscopist, while $0.5 \mathrm{mg}$ nitroglycerin tablets or normal placebo tablets would be administered under the tongue $5 \mathrm{~min}$ before the operation.
The technique for the placement of the prophylactic pancreatic stent and the type of stent used were not directed by the study protocol but were instead left to the discretion of the treating endoscopist. This approach was intended to mimic real-world practice, wherein variations in stent type, caliber, and length exist. After the operation, somatostatin was injected intravenously through a micropump at $250 \mu \mathrm{g} / \mathrm{h}$ for 12 to $48 \mathrm{~h}$. When the blood amylase level was normal, the medication was stopped. Nitroglycerin or normal saline placebo was added to a normal saline drip for $6 \mathrm{~h}$ after the operation at a rate of $10 \mu \mathrm{g} /(\mathrm{kg} \cdot \mathrm{min})$. In cases of severe hypotension or severe headache, the medication was withdrawn. As resistin level is significantly related to the severity of pancreatitis, the concentration of serum resistin was determined using an enzyme-linked immunosorbent assay (ELISA) kit (Market Inc., Company, United States) in order to evaluate the severity of PEP more accurately.

Since the endoscopist(s), endoscopy nurse, and technician/assistant who performed the ERCP procedure were aware of whether or not a stent was placed, these individuals were not involved in the post-operative care of the patients for at least $48 \mathrm{~h}$, at which point the of PEP, as the primary endpoint, was apparent. This approach was critical to maintaining blinding (of patients, treating clinical personnel, and outcome adjudicators) to ensure equal co-interventions among the three groups and unbiased adjudication of the primary outcome. Additionally, the placement of a stent was not recorded in the patients' endoscopy reports or medical records.

The patients were followed-up on days 5 and 30 after ERCP. The goal of the first follow-up was to ascertain the data necessary to adjudicate the primary endpoint. The goal of the second follow-up was to ascertain the data necessary to adjudicate the secondary outcome and to assess the patients for delayed serious adverse events.

The levels of serum resistin were assayed using commercial ELISA kits, according to the manufacturer's instructions (Ray Biotech, USA).

\section{Indicators}

The occurrence of abdominal pain, nausea, vomiting, and other symptoms after the procedure, as well as serum amylase and resistin levels before and after were observed and recorded. The diagnosis and severity grading of PEP were carried out according to the revised Atlanta classification (21). Both incidence of pancreatitis and hyperamylasemia following ERCP was compared between 
Table 1 Demographic characteristics of the 526 patients who underwent endoscopic retrograde cholangiopancreatography

\begin{tabular}{lccccc}
\hline Variable & Mean \pm SD & $95 \%$ Cl & Me & Min. & Max. \\
\hline Age & $65.5 \pm 13.2$ & $(63.95,67.16)$ & 65 & 35 & 258 \\
AST & $105.7 \pm 50.1$ & $(99.66,111.84)$ & 105 & 16 & 11 \\
ALT & $119.5 \pm 52.9$ & $(113.09,125.96)$ & 119 & 39 \\
ALP & $173 . \pm 18.5$ & $(171.12,175.63)$ & 173 & 381 \\
Amylase & $91.59 \pm 13.1$ & $(89.99,93.19)$ & 91 & 30 & 110 \\
\hline
\end{tabular}

$\mathrm{Cl}$, confidence interval; Me, median; Min., minimum; Max., maximum; AST, glutamic pyruvic transaminase; ALT, glutamic oxaloacetic transaminase; ALP, alkaline phosphatase.

the three groups. Adverse reactions to nitroglycerin and indomethacin, including hypotension and headache, were evaluated.

\section{Statistical analysis}

Data were analyzed with SPSS version 19 (SPSS, Inc., Chicago, IL, USA). Values were analyzed with analysis of variance (ANOVA), $t$-tests, and Fisher's exact tests. The difference between groups is represented by Fisher's T exact tests and one-way analysis of variance. All $\mathrm{P}$ values were two-sided, and $\mathrm{P}<0.05$ was considered statistically significant.

\section{Results}

A total of 526 patients were eligible for inclusion and were divided into the placebo group, the indomethacin + nitroglycerin group, and the PSP group. The placebo group included 176 patients with a mean age of $63.5 \pm 14$.4 range, $35-76$ years. The mean number of stones was $2.7 \pm 1.0$ range, $1-6$, and the mean common bile duct (CBD) diameter was $9.71 \pm 3.90 \mathrm{~mm}$ range, $5-16 \mathrm{~mm}$.

The indomethacin + nitroglycerin group included 176 patients with a mean age of $66.87 \pm 13.04$ (range, 40-77) years. The mean number of stones was $2.6 \pm 0.9$ (range, 1-6) and the mean CBD diameter was 9.94 $\pm 4.14 \mathrm{~mm}$ (range, 5-16 mm).

The PSP group included 174 patients with a mean age of $66.30 \pm 12.00$ (range, $40-80$ ) years. The mean number of stones was $2.8 \pm 1.0$ (range, 1-6) and the mean CBD diameter was $9.81 \pm 4.25 \mathrm{~mm}$ (range, $5-16 \mathrm{~mm}$ ). There was no significant differences in age, average stone count, or CBD diameter between the three groups $(\mathrm{P}>0.05)$.

Seventy four patients were excluded due to the use of drugs that interfered with the treatment during the study period or for other reasons, such as failure to complete ERCP for duodenal papillary lesions. The primary outcome of the study was PEP and the secondary outcome was the severity of PEP. The study outcomes were independently assessed by three adjudicators based on a review of the patients' adverse event and medical records after ERCP.

There was no significant difference between the three groups in terms of age, risk of PEP, or ERCP procedure time $(\mathrm{P}>0.05)$. The demographic and clinical characteristics of the patients are shown in Tables 1 and 2.

\section{Incidence of postoperative pancreatitis}

The incidence of PEP was $19.3 \%, 5.1 \%$ and $12.1 \%$ in placebo group, the indomethacin + nitroglycerin group, and the PSP group, respectively, which represented a significant difference between all three groups $(\mathrm{F}=6.445$, $\mathrm{P}=0.011$ ). A significant difference was found between groups indomethacin + nitroglycerin group and PSP group (Pearson $=4.551, \mathrm{P}=0.037)$. In the three groups, there were 14,5 , and 13 cases of mild pancreatitis, respectively, and 20, 4, and 8 cases of moderate pancreatitis, respectively. There was a significant difference between the incidence of mild PEP in indomethacin + nitroglycerin group and PSP group (Pearson $=11.086, \mathrm{P}=0.002$ ), and the two groups also showed a significant difference in the incidence of moderate PEP (Fisher $=5.602, \mathrm{P}=0.027$ ). No significant differences were found between the groups for the other risk factors, including cannulation time, pre-cut sphincterotomy, and the duration of somatostatin treatment. All patients who developed pancreatitis after ERCP continued fasting and their conditions improved after routine acid inhibition, enzyme inhibition, and anti-infection treatment (Table 2). 
Table 2 Endoscopic retrograde cholangiopancreatography characteristics and complications

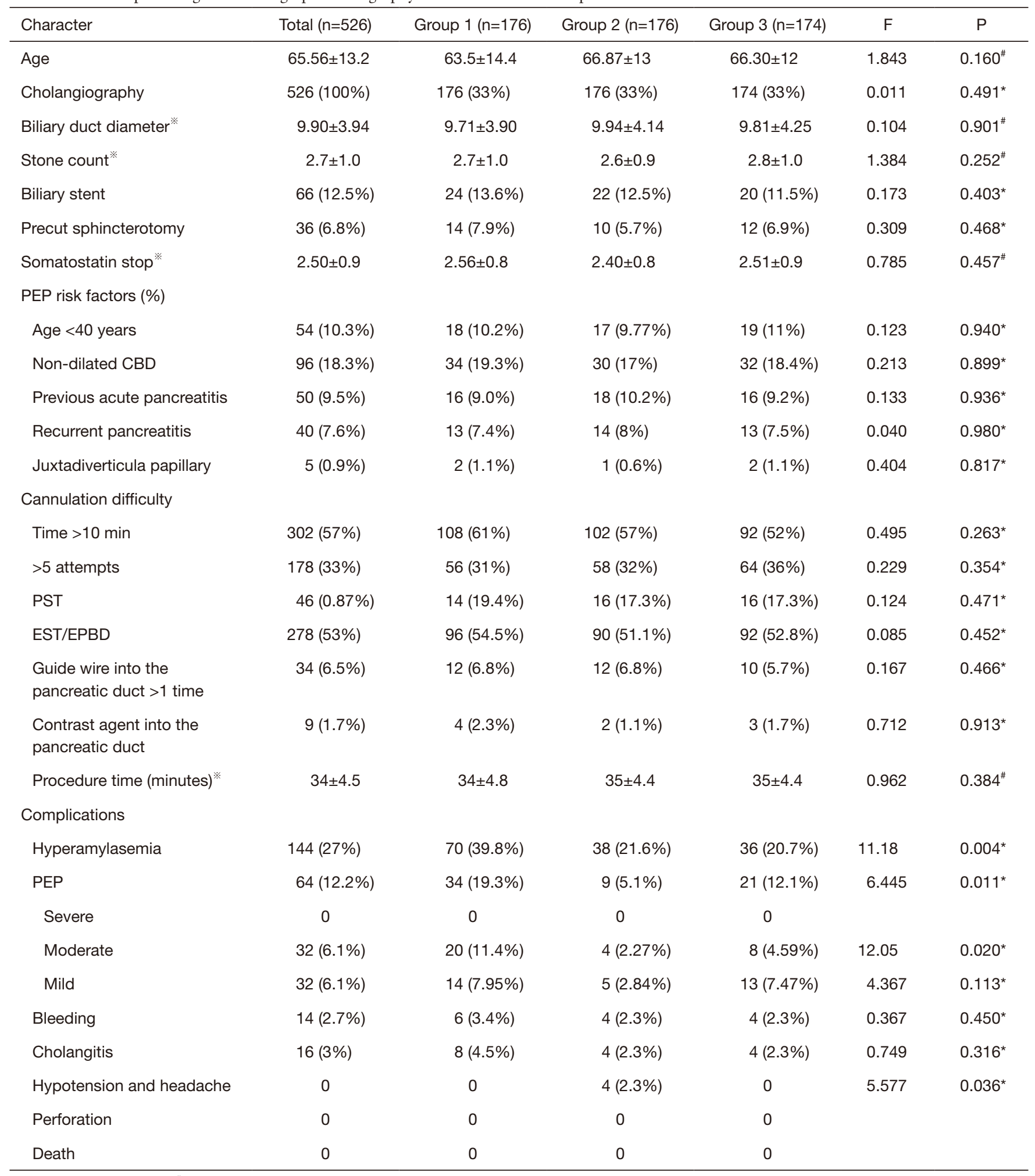

*, (mm; mean \pm SD); ", ANVOA test; *, Fisher's exact test. PEP, post-endoscopic retrograde cholangiopancreatography pancreatitis; CBD, common bile duct; PST, pre-cut sphincterotomy; EST/EPBD, endoscopic sphincterotomy/balloon dilatation. 
Table 3 Comparison of serum amylase levels of the patients before and after endoscopic retrograde cholangiopancreatography in the three groups $(\mathrm{U} / \mathrm{L})$

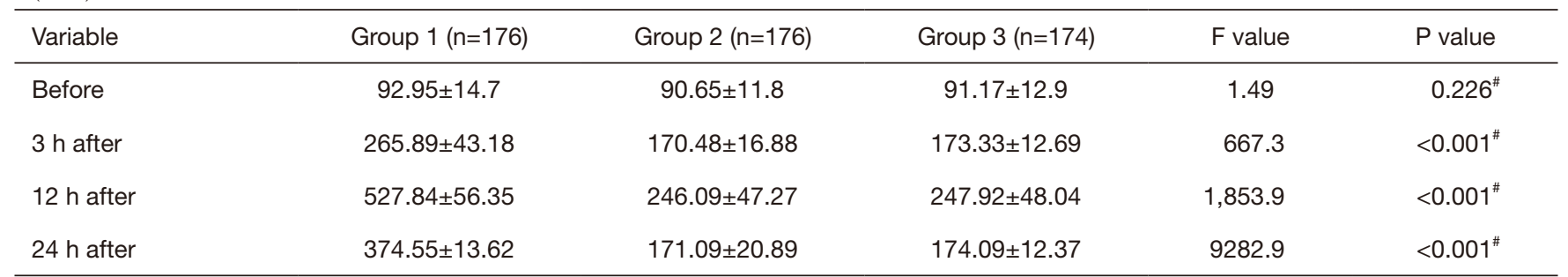

\#, ANVOA test.

Table 4 Changes in serum resistin levels $\left(\mu \mathrm{g} \cdot \mathrm{L}^{-1)}\right.$ in each group

\begin{tabular}{|c|c|c|c|c|c|}
\hline Variable & Group $1(n=176)$ & Group $2(n=176)$ & Group $3(n=174)$ & $\mathrm{F}$ value & $P$ value \\
\hline $3 d$ & $259.02 \pm 35.5$ & $111.67 \pm 27.2^{\mathrm{a}}$ & $110.76 \pm 28.7$ & $1,344.413$ & $<0.001^{\#}$ \\
\hline $7 d$ & $175.87 \pm 21.4$ & $43.10 \pm 8.25$ & $144.51 \pm 8.52$ & $5,117.858$ & $<0.001^{\#}$ \\
\hline
\end{tabular}

", ANVOA test.

\section{Incidence of postoperative hyperamylasemia in groups placebo, indomethacin + nitroglycerin, and PSP}

The incidence of postoperative hyperamylasemia in the placebo group, the indomethacin + nitroglycerin group and the PSP group was $39.8 \%, 21.6 \%$, and $20.7 \%$, respectively. No significant difference existed between the incidence of postoperative hyperamylasemia in indomethacin + nitroglycerin group and PSP group $(\mathrm{P}=0.525)$; however, the incidences in both groups were significantly lower than that in the placebo group $(\mathrm{P}=0.039$ and $\mathrm{P}=0.031$ for indomethacin + nitroglycerin group and PSP group, respectively). All patients who developed hyperamylasemia postoperatively continued fasting and underwent conventional acid inhibition, enzyme inhibition, and antiinfection treatment (Table 3).

\section{Resistin levels before and after ERCP}

There was no significant difference between the levels of resistin in the indomethacin + nitroglycerin group and the PSP group on day 1 or 3 ( $\mathrm{t}=0.272$ and $\mathrm{P}=0.787$; and $\mathrm{t}=1.060$ and $\mathrm{P}=0.292$, respectively). However, on day 7 , a significant difference was observed between these two groups ( $\mathrm{t}=52.510$, $\mathrm{P}<0.001)$. Table 4 shows the changes in the levels of resistin in the three study groups.

\section{Adverse events}

In terms of adverse reactions, four patients in the indomethacin + nitroglycerin group experienced serious hypotension and headache (Fisher $=5.577, \mathrm{P}=0.036$ ). The characteristics and complications of ERCP in the three study groups.

\section{Discussion}

A large number of studies have suggested that oral nitroglycerin and rectal indomethacin can reduce the risk of PEP in patients who are considered to be at high risk preoperatively. Moreover, as a cheaper alternative to PSP, this treatment could also help to reduce patients' medical expenses. However, to the best of our knowledge, no study had compared the effectiveness of this drug combination with that of PSP in preventing PEP in patients with difficult intubation. Therefore, we designed a clinical trial to investigate this.

The majority of cases of PEP are mild and uncomplicated; however, severe pancreatitis accounts for up to $30 \%$ of cases (22-25). In the current study, all patients presented with mild or moderate pancreatitis, and there was no clinical evidence of severe pancreatitis occurring.

Indomethacin reduce the inflammatory response (26), 


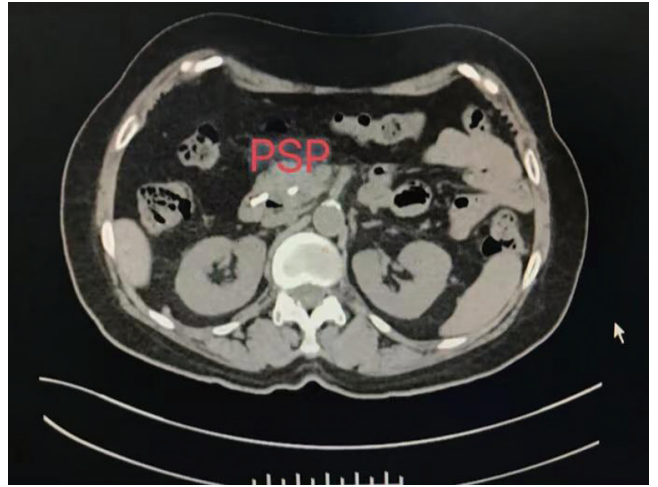

Figure 2 Representative CT images after PSP treatment 1. CT, computed tomography; PSP, pancreatic stent placement.

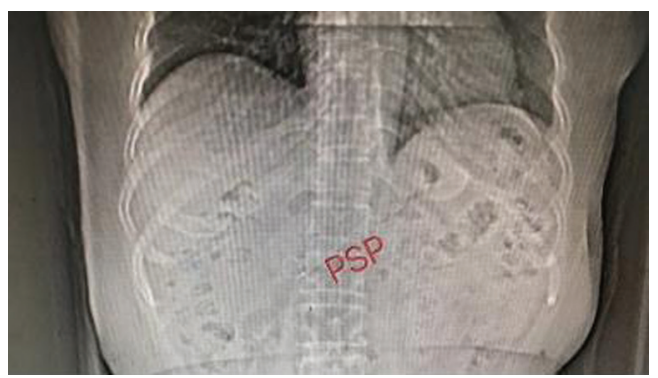

Figure 3 Representative CT images after PSP treatment 2. CT, computed tomography; PSP, pancreatic stent placement.

and nitroglycerin can reduce the pressure on the sphincter of Oddi (27). In this study, the two drugs were prophylactically administered to patients undergoing ERCP, and their preventive effects on pancreatitis and hyperamylasemia were observed. Under the same set of treatment factors, no significant difference was found in the incidence of hyperamylasemia in the indomethacin + nitroglycerin group, and the PSP group. However, the incidences of PEP were lower than that in the placebo group, and the incidence of PEP in group the indomethacin + nitroglycerin group was lower than that in group the PSP group. Moreover, the grading of severity of PEP in the indomethacin + nitroglycerin group was lower than that in group the PSP group (Figures 2 and 3).

For more accurate detection of the occurrence of PEP in this study, the concentration of resistin was measured. Previous studies have shown that resistin concentration is significantly increased in severe acute pancreatitis, and resistin is expected to become a novel marker for evaluating the severity of acute pancreatitis (28-30). The results in this study showed that the concentration of resistin increased significantly on the first day of PEP and decreased gradually after ERCP 3 days. The change in resistin concentration was related to the condition of the PEP patients; the change in group 1 was greater than those in the indomethacin + nitroglycerin group and the PSP group. On day 7 after ERCP, the levels of resistin in the indomethacin + nitroglycerin group showed a more rapid decrease than those in the PSP group, which indicated a significant reduction in pancreatic inflammation. If an adverse event occurs during the experiment, we will quickly investigate the serious adverse event, take necessary measures to ensure the safety and rights of the subjects, and promptly report to the drug supervision and management department and the health administration department, and to the same drug Other investigators of the clinical trials.

The application of pancreatic duct stents to prevent PEP is considered to be effective or possibly effective in most current studies. In this study, there was significant difference in the incidence of PEP and the grading of severity between the indomethacin + nitroglycerin group and the PSP group. Drug safety of indomethacin + nitroglycerin was controllable, but drug administration resulted in less trauma and greater maneuverability. The prophylactic application of indomethacin + nitroglycerin has a certain protective effect against PEP. However, large-sample clinical trials are needed to further investigate and confirm this effect.

Indomethacin + nitroglycerin offers several additional advantages over PSP. First, it avoids the phenomenon of failed PSP, which is associated with a high rate of PEP. It also eliminates the risk of significant non-pancreatitis adverse events induced by PSP, such as stent migration and duct perforation. Moreover, treatment with indomethacin + nitroglycerin can substantially reduce healthcare expenses by eliminating the cost of stent placement in most cases, as well as eliminating the need for follow-up abdominal radiography and upper endoscopy to remove stents.

In conclusion, the preoperative application of indomethacin + nitroglycerin can prevent and relieve the severity of PEP after ERCP in patients with difficult intubation. However, more high-quality studies are needed in the future to further confirm the effectiveness of this drug combination and to provide new ideas for the clinical application of drugs for PEP prevention.

\section{Acknowledgments}

Funding: We acknowledge the work of fund Supported by 
Key Disciplines Construction Project of Pudong Health Bureau of Shanghai (No. PWZxk2017-11) and Shanghai Municipal Health and Family Planning Commission Scientific Research Project Program (201740295).

\section{Footnote}

Reporting Checklist: The authors have completed the CONSORT reporting checklist. Available at http://dx.doi. org/10.21037/apm-20-1963

Data Sharing Statement: Available at http://dx.doi. org/10.21037/apm-20-1963

Conflicts of Interest: All authors have completed the ICMJE uniform disclosure form (available at http://dx.doi. org/10.21037/apm-20-1963). The authors have no conflicts of interest to declare.

Ethical Statement: The authors are accountable for all aspects of the work in ensuring that questions related to the accuracy or integrity of any part of the work are appropriately investigated and resolved. All procedures performed in this study involving human participants were in accordance with the Declaration of Helsinki (as revised in 2013). The study was approved by Ethics Committee of the Pudong New Area People's Hospital, Shanghai University of Medicine \& Health Sciences (Shanghai, China) (No. 2017-26). Written informed consent was obtained from all patients.

Open Access Statement: This is an Open Access article distributed in accordance with the Creative Commons Attribution-NonCommercial-NoDerivs 4.0 International License (CC BY-NC-ND 4.0), which permits the noncommercial replication and distribution of the article with the strict proviso that no changes or edits are made and the original work is properly cited (including links to both the formal publication through the relevant DOI and the license). See: https://creativecommons.org/licenses/by-nc-nd/4.0/.

\section{References}

1. Kang X, Zheng L, Zeng W, et al. Risk Factors for PostERCP Pancreatitis in High-Risk Patients Receiving Postprocedure Rectal Indomethacin. J Gastrointest Surg 2018;22:1903-10.

2. Park CH, Jung JH, Hyun B, et al. Safety and efficacy of early feeding based on clinical assessment at 4 hours after ERCP: a prospective randomized controlled trial. Gastrointest Endosc 2018;87:1040-9.el.

3. Clores MJ, Bucobo JC, D'Souza LS, et al. Radiopaque Short Pancreatic Stents Reliably Migrate in Nearly All Patients When Inserted for Prevention of Pancreatitis. Clin Clin Gastroenterol Hepatol 2020;18:739-40.

4. Sugimoto M, Takagi T, Suzuki R, et al. Pancreatic stents for the prevention of post-endoscopic retrograde cholangiopancreatography pancreatitis should be inserted up to the pancreatic body or tail. World J Gastroenterol 2018;24:2392-9.

5. Pérez Roldán F, González Carro P. Pancreatic stents in ERCP. Where are we? Rev Esp Enferm Dig 2018;110:413-5.

6. Fujisawa T, Kagawa K, Ochiai K, et al. Prophylactic Efficacy of 3- or 5-cm Pancreatic Stents for Preventing Post-ERCP Pancreatitis: A Prospective, Randomized Trial. J Clin Gastroenterol 2016;50:e30-4.

7. $\mathrm{Lu} \mathrm{Y,} \mathrm{Jin} \mathrm{Z,} \mathrm{Wu} \mathrm{JC,} \mathrm{et} \mathrm{al.} \mathrm{Endoscopic} \mathrm{retrieval} \mathrm{technique}$ of proximally migrated pancreatic stents: a retrospective study in a tertiary centre. Gastroenterol Res Pract 2015;2015:485980.

8. Nagashima K, Ijima M, Kimura K, et al. Does the Use of Low Osmolality Contrast moderate Reduce the Frequency of Post-Endoscopic Retrograde Cholangiopancreatography Pancreatitis: A Comparative Study between Use of Low and High Osmolality Contrast Media. Digestion 2019;26:1-6.

9. Geraci G, Palumbo VD, D'Orazio B, et al. Rectal Diclofenac administration for prevention of postEndoscopic Retrograde Cholangio-Pancreatography (ERCP) acute pancreatitis. Randomized prospective study. Clin Ter 2019;170:e332-6.

10. Lyu Y, Wang B, Cheng Y, et al. Comparative Efficacy of 9 Major Drugs for Postendoscopic Retrograde Cholangiopancreatography Pancreatitis: A Network Meta-Analysis. Surg Laparosc Endosc Percutan Tech 2019;29:426-32.

11. Sotoudehmanesh R, Ali-Asgari A, Khatibian M, et al. Pharmacological prophylaxis versus pancreatic duct stenting plus pharmacological prophylaxis for prevention of post-ERCP pancreatitis in high risk patients: a randomized trial. Endoscopy 2019;51:915-21.

12. Pekgöz M. Post-endoscopic retrograde cholangiopancreatography pancreatitis: A systematic review for prevention and treatment. World J Gastroenterol 2019;25:4019-42. 
13. Radadiya D, Devani K, Arora S, et al. Peri-Procedural Aggressive Hydration for Post Endoscopic Retrograde Cholangiopancreatography (ERCP) Pancreatitis Prophylaxsis: Meta-analysis of Randomized Controlled Trials. Pancreatology 2019;19:819-27.

14. Avila P, Holmes I, Kouanda A, et al. Practice patterns of post-ERCP pancreatitis prophylaxis techniques in the United States: a survey of advanced endoscopists. Gastrointest Endosc 2020;91:568-73.e2.

15. Serrano JPR, de Moura DTH, Bernardo WM, et al. Nonsteroidal anti-inflammatory drugs versus placebo for post-endoscopic retrograde cholangiopancreatography pancreatitis: a systematic review and meta-analysis. Endosc Int Open 2019;7:E477-86.

16. Lopes L, Canena J. ERCP in Portugal. A Wide Survey on the Prevention of Post ERCP Pancreatitis and Papillary Cannulation Techniques.GE Port J Gastroenterol 2018;26:14-23.

17. Liu L, Li C, Huang Y, et al. Nonsteroidal Antiinflammatory Drugs for Endoscopic Retrograde Cholangiopancreatography Postoperative Pancreatitis Prevention: a Systematic Review and Meta-analysis. J Gastrointest Surg 2019;23:1991-2001.

18. Tomoda T, Kato H, Mizukawa S, et al. A Multicenter, Prospective, Randomized Controlled Trial Evaluating the Efficacy of Rectal Diclofenac and Sublingual Nitroglycerin as a Combined Prophylactic Treatment for Post-ERCP Pancreatitis. Acta Med Okayama 2016;70:405-8.

19. Dumonceau JM, Andriulli A, Elmunzer BJ, et al. European Society of Gastrointestinal Endoscopy. Prophylaxis of post-ERCP pancreatitis: European Society of Gastrointestinal Endoscopy (ESGE) Guideline - updated June 2014. Endoscopy 2014;46:799-815.

20. Shao LM, Chen QY, Chen MY, et al. Nitroglycerin in the prevention of post-ERCP pancreatitis: a meta-analysis. Dig Dis Sci 2010;55:1-7.

21. Smeets X, Bouhouch N, Buxbaum J, et al. The revised Atlanta criteria more accurately reflect severity of post-

Cite this article as: Wang $\mathrm{Y}, \mathrm{Xu} \mathrm{B}$, Zhang $\mathrm{W}$, Lin $\mathrm{J}, \mathrm{Li}$ G, Qiu W, Wang Y, Sun D, Wang Y. Prophylactic effect of rectal indomethacin plus nitroglycerin administration for preventing pancreatitis after endoscopic retrograde cholangiopancreatography in female patients. Ann Palliat Med 2020;9(6):4029-4037. doi: 10.21037/apm-20-1963
ERCP pancreatitis compared to the consensus criteria. United European Gastroenterol J 2019;7:557-64.

22. Shimamura T, Miyahara K, Takamori A, et al. Risk Factors for Post-Endoscopic Retrograde Pancreatography Pancreatitis: A Retrospective Chart Review in a Regional Hospital in Japan. Digestion 2020;101:557-62.

23. Syrén E, Eriksson S, Enochsson L, et al. Risk factors for pancreatitis following endoscopic retrograde cholangiopancreatography. BJS Open 2019;3:485-9.

24. Perdigoto DN, Gomes D, Almeida N, et al. Risk Factors for Post-Endoscopic Retrograde Cholangiopancreatography Pancreatitis in the Indomethacin Era-A Prospective Study. GE Port J Gastroenterol 2019;26:176-83.

25. Lai JH, Hung CY, Chu CH, et al. A randomized trial comparing the efficacy of single-dose and double-dose administration of rectal indomethacin in preventing post-endoscopic retrograde cholangiopancreatography pancreatitis. Medicine (Baltimore) 2019;98:e15742.

26. Li L, Han Z, Yuan H, et al. Nonsteroidal antiinflammatory drugs reduce the incidence of postendoscopic retrograde cholangio- pancreatography pancreatitis: a meta-analysis. J Hepatobiliary Pancreat Sci 2017;24:520-9.

27. Katsinelos P, Lazaraki G, Chatzimavroudis G, et al. Impact of nitroglycerin and glucagon administration on selective common bile duct cannulation and prevention of postERCP pancreatitis. Scand J Gastroenterol 2017;52:50-5.

28. Yu P, Wang S, Qiu Z, et al. Efficacy of resistin and leptin in predicting persistent organ failure in patients with acute pancreatitis. Pancreatology 2016;16:952-7.

29. Xue LN, Wang XY, Tan Y, et al. Significance of resistin expression in acute pancreatitis. Exp Ther Med 2015;9:1438-42.

30. Karpavicius A, Dambrauskas Z, Gradauskas A, et al. The clinical value of adipokines in predicting the severity and outcome of acute pancreatitis. BMC Gastroenterol 2016;16:99. 